\title{
Psychopathy and Associated Factors Among Newly Admitted Prisoners in Correctional Institution Located in Bench Sheko and West Omo Zone, South West Ethiopia: A Cross-Sectional Study
}

This article was published in the following Dove Press journal: Psychology Research and Behavior Management

\author{
Asrat Wolde' \\ Yonas Tesfaye $\mathbb{D D}^{2}$ \\ Yimenu Yitayih (1D) ${ }^{2}$ \\ 'Department of Psychiatry, Colleague of \\ Medicine and Health Sciences, Mizan Tepi \\ University, Mizan Aman, Ethiopia; \\ ${ }^{2}$ Department of Psychiatry, Faculty of \\ Medical Sciences, Jimma University, \\ Jimma, Ethiopia
}

Background: Psychopathy is an emerging health and behavioral problem worldwide. Psychopathy is linked to risk substance use, maltreatment, violence, crime, and reoffending, but little is known about psychopathy in low income countries like Ethiopia. Therefore, we assessed the prevalence and factors associated with psychopathy among newly-admitted prisoners in Bench Sheko and West Omo zone correctional center, Mizan Aman, Ethiopia.

Methods: A cross-sectional study was conducted from April 20 to July 19, 2019, among 411 ( N=422) newly-admitted Bench Sheko and West Omo zone correctional center prisoners by using a consecutive sampling technique. A semi-structured and interviewer administered psychopathy checklist revised tool was used for screening psychopathy. WHO, ASSIST tool was used for screening risk use of khat, tobacco, and alcohol. Trauma, maltreatment, and social support were assessed with a life event checklist, adverse life experience screening tool, and Oslo social support scale, respectively. In addition, Criminal and clinical history of the prisoner was also assessed. The data was entered into Epi-data 3.1 and exported to Statistical Package for Social Science (SPSS) Version 21 for statistical analysis. A logistic regression model was used. Variables with a $p$-value less than 0.05 in the final fitting model were declared as independent predictors of psychopathy.

Results: The prevalence of psychopathy was $24.3 \%$. Childhood maltreatment (AOR=6, 95\% $\mathrm{CI}=2.2-17.5)$, risky khat use $(\mathrm{AOR}=4.6,95 \% \mathrm{CI}=2.4-8.7)$, poor social support $(\mathrm{AOR}=3.5$, 95\% CI=1.9-6.6), family history of imprisonment ( $\mathrm{AOR}=3,95 \% \mathrm{CI}=1.5-6)$, history of trauma $(\mathrm{AOR}=2.3,95 \% \mathrm{CI}=1.1-4.8)$, and reoffending $(\mathrm{AOR}=2,95 \% \mathrm{CI}=1.1-3.8)$ were positively associated variables with psychopathy.

Conclusion: Psychopathy is highly prevalent among newly-admitted prisoners. Integrated efforts involving relevant stakeholders are needed to design strategies for early screening of psychopathy to prevent reoffending, and management of risk substance use at admission is crucial.

Keywords: psychopathy, prisoners, crime, khat use, Ethiopia

\section{Background}

Department of Psychiatry, Colleague of Medicine and Health Sciences, Mizan Tepi University, Mizan Aman, Ethiopia

Tel +251916389179

Email Asratwolde2017@gmail.com
Psychopathy as a severe form of antisocial personality disorder is a serious mental health disorder with social, emotional, and behavioral impairment. ${ }^{1,2}$ Psychopathy is known by impulsivity, incentive seeking behavior, violence, persistent violation 
of social norms, and the inability to experience empathy and remorse. Psychopathy co-occurs with a number of life threatening behaviors: including risk substance use, early life trauma exposure, and physical abuse..$^{3-6}$ Individuals with psychopathy are among the most dangerous and chronic offenders, as evidenced by high recidivism rates. ${ }^{7,8}$ Psychopathic personalities are linked to prohibited behavior and problematic substance use, and substance use greatly increases the possibility that psychopathic individuals will be involved in serious or violent criminal activity. ${ }^{9}$ Psychopathy is caused by a combination and interaction of hereditary and environmental influences. ${ }^{10}$ People with psychopathy have decreased activity in the prefrontal cortex and have difficulty in behavior control, planning in advance, and determining the value of their action. $^{11}$ A study shows that $14.7 \%$ of prisoners were positive for psychopathy, and psychopathy is associated with recidivism, depression, anxiety, and reactive and proactive aggression. ${ }^{12}$ Having any parent incarcerated during childhood can be a risk factor for psychopathy for their children. ${ }^{13}$ The study officially reported that violent misconduct in prison independently contributed to psychopathy and impulsivity, ${ }^{14}$ and having adequate social support is protective against psychopathy. ${ }^{15}$ A considerable portion (37-93\%) of the effects of childhood maltreatment was the risk for psychopathic personality traits. ${ }^{16}$ The meta-analysis from 29 unique samples from 22 studies disclosed that the mean psychopathy score was $21.2 \%$, and psychopathic personality is a major risk factor for various forms of lethal violence and recidivism. ${ }^{17}$ Psychopathic personality trait is associated with younger age, male gender, suicide attempts, imprisonment, homelessness, personality disorder (histrionic, obsessive compulsive, borderline, and antisocial), and panic disorder. ${ }^{18}$ The majority of offenders (58-97\%) who committed sexual murder meet the criteria for psychopathy, as well as being motivated by revenge. ${ }^{19}$ Psychopathic offenders were about 5-times more likely than other offenders to violently reoffend within 5 years of discharge. ${ }^{20}$ In addition, psychopathy was a vigorous forecaster of general, violent, and sexual recidivism, and about $80 \%$ of psychopathic offenders committed a new violent offense within 1 -year of release. ${ }^{21}$ Many studies show group and individual insight oriented psychotherapy was intervened for psychopathic individuals and was effective. ${ }^{22,23}$ Despite the importance of psychopathy for criminal behavior, there is a lack of information about psychopathy and its effect among prisoners in our country, Ethiopia. Therefore, the aim of this study was to assess the prevalence and factors associated with Psychopathic personality among newly-admitted prisoners in Bench Sheko and West Omo zone correctional center, Mizan Aman, Ethiopia.

\section{Methods}

\section{Study Setting and Period}

The study was conducted in Bench Sheko and West Omo zone correctional center from April 20 to July 19, 2019. Bench Sheko and West Omo zone is located in Southern Nation Nationality and Peoples Regional State, which is $565 \mathrm{~km}$ from the capital city of Ethiopia, Addis Ababa. It has a population of 781,006 according to the 2009 census. It was established in 1973. It gives service for Bench Sheko and West Omo zone and its surrounding zones. The correctional center had 2,025 sentenced prisoners at the time of study. It has one clinic serving prisoners, with no mental health service.

\section{Study Design}

Institution-based cross-sectional study design was conducted.

\section{Population}

\section{Source Population}

All newly admitted prisoners in Ethiopia.

\section{Study Population}

All newly admitted prisoners who fulfill the eligibility criteria at the time of data collection period.

\section{Eligibility Criteria}

Inclusion Criteria

All newly admitted prisoners who arrived during the data collection period were included in the study.

\section{Exclusion Criteria}

Having hearing and communication impairment, those who are acutely sick, and those aged less than 18 years were excluded.

\section{Sample Size Determination and Sampling Technique Sample Size Determination}

The sample size (n) was calculated by using the single population proportion formula, $\mathrm{n}=((\mathrm{Z}$ alpha/2 $) 2 \times \mathrm{p}(1-\mathrm{p})) /$ $\mathrm{d} 2$, assuming a prevalence $(\mathrm{p})=50 \%$, because we could not find a similar study done in our country or near to our 
country. Using a 95\% confidence interval (CI) of $1.96(\mathrm{Z}$ alpha/2=1.96), $(d=0.05)$, and a non-response rate of $10 \%$, the final sample size was 422 .

\section{Sampling Technique}

A consecutive sampling technique was used. Every newly admitted prisoner was interviewed until the required sample size was saturated.

\section{Procedure}

Data was collected using a pre-tested semi-structured questionnaire, which has five parts: Socio-demographic, environmental, clinical, substance, criminal factors, and PCL-R. A questionnaire for psychopathy, Hare's PCL-R, was used to assess for the presence of psychopathy, which has two factor designs: Factor one reflects affective symptoms and factor two relates to antisocial behavior. It is a gold standard tool used throughout the world and scored based on interview, file review, and collateral information.

The PCL-R has a 20-item symptom score scale. Each item in the psychopathy checklist revised is measured on three points $(0,1$, and 2$)$. According to specific criteria, 0 is assigned if the item does not apply, 1 is assigned if it applies somewhat, and 2 is assigned if it applies fully. PCL-R has a total score of 40. The PCL-R score of 30 and above were considered as positive for psychopathy. ${ }^{2}$ The reliability of item and inter-rater reliability were $\alpha=0.87$ and 0.91 , respectively. The Cronbach's alpha in this study was 0.92 .

The questionnaire was used to assess explanatory variables for psychopathy. Socio-economic factors, environmental factors (social support, childhood maltreatment, traumatic life event, excessive childhood or adolescent television viewing, and family conflict), clinical factors (history of known mental and chronic medical illness, family history of mental illness, family history of alcohol use), and criminal factors (reconviction, family history of prison). Social support was assessed by using the Oslo-3 social support scale. ${ }^{24}$ Substance use was assessed by the World Health Organization, Alcohol, Smoking, and Substance Involvement Screening Test (ASSIST). ${ }^{25}$ A life event checklist was used for traumatic life events, ${ }^{26}$ and an adverse life experience tool questionnaire was used for childhood maltreatment. ${ }^{27}$ These variables were used because studies have found that they are pertinent for psychopathy.

Data collectors were masters' psychiatric nurses working in Mizan Tepi University teaching hospital.
Supervisors were clinical mental health specialists. Training was given for data collectors and supervisors by the principal investigator on methods of data collection and ethical issues. In addition, training was given on the psychopathy checklist revised tool for data collectors and supervisors by a psychiatrist trained in the use of the tool on how to interview and score it. PCL-R score was assessed by both interview and a file review. Data collectors conducted the psychopathy checklist revised assessment interviews after carrying out test assessment interviews in the presence of a trainer to establish standards for scoring individual PCL-R items. Moreover, other tools used in this study were clearly explained for data collectors and supervisors by the principal investigator. The supervisor was checking the completeness of the collected data daily. Data was collected using a face-toface interview by structured Amharic version questionnaire. The English version questionnaire was translated to Amharic version, and then it was translated back to English by an independent language expert person to check for consistency.

A pretest was conducted on $5 \%$ of the sample size 1 week prior to the actual data collection, on kefa zone prison center, which is located $112 \mathrm{~km}$ away from Mizan Aman town, to identify potential problems in data collection tools. Correction of the questionnaire (grammatical errors and local language translation discrepancy) which might affect the findings negatively were corrected according to the pretest result.

\section{Data Analysis}

The entire questionnaire was checked for completeness manually. The data was cleaned, coded and entered in the computer using Epi-Data version 3.1, then it was exported to SPSS 21 version statistical software for analysis. Characteristics of respondents were analyzed by descriptive statistics using tables and frequency.

Bivariate and multivariate logistic regressions were used to identify the independent factors for psychopathy and crude and adjusted odds ratio was computed for each variable to determine the strength of association. A bivariate analysis was performed to assess the association of each independent factor with psychopathy. Thena candidate variable with a $p$-value less than 0.25 on bivariate analysis was entered into multivariate logistic regression to control the con-founder after checking the multicollinearity. The assumption fitness was tested by Hosmer Lemeshow goodness fit test. Independent factors 
associated with psychopathy were declared at a $p$-value $<0.05$ cut-off point and strength of the association was assessed using AORs with their corresponding CIs at $95 \%$. Then, a variable with a $p$-value less than 0.05 on multivariable logistic regression was considered as statistically significant.

\section{Ethical Consideration}

This study was conducted in accordance with the Declaration of Helsinki. Ethical clearance was obtained from the Review Board of Jimma University. The purpose of the study was informed for the study participants. Permission was obtained from prison administration. Written consent was obtained from all study participants before interview. Participation in the study was completely voluntary, and the right to withdraw from the interview was also secured. Confidentiality of the information was ensured throughout the study.

\section{Results}

\section{Socio-Demographic and Economic Characteristics}

A total of 411 prisoners participated in this study, with the response rate of $97.4 \%$. The mean age of the participants' age in a year was $29.24 \pm 8.33 \mathrm{SD}$ and more than half (249, $60.4 \%$ ) of the participants were in the age groups $25-$ 44 years. Regarding the sex of the respondents, almost all $(98.3 \%)$ were males and nearly two thirds $(265,65.2 \%)$ of the participants were protestant in religion. Concerning toethnicity, half $(208,50.6 \%)$ were Bench, followed by Shako $(63,15.3 \%)$. Concerning their marital status, nearly half $(227,55.2 \%)$ were married and about two thirds $(273$, $66.4 \%$ ) of the participants completed their primary education. Regarding their occupation, 210 (51.1) and 83 (20.2\%) were farmers and daily laborers, respectively. The majority $(349,84.4 \%)$ had an average monthly income greater than 1,200 Ethiopian birr (see Table 1).

\section{Prevalence of Psychopathy}

The prevalence of psychopathy was 100 (24.3\%) from the total participants. Among the reconvicted prisoners, 88 $(69 \%)$ were psychopathic. From the total psychopathic prisoners, $60(60 \%)$ committed homicide crime. From the total of 100 psychopathy participants, half of them had a positive family history of imprisonment. Out of the participants with psychopathy, $62(62 \%)$ used khat in the past 3 months before their current incarceration.

\section{Criminal and Prison-Related Factors}

All of the prisoners were convicted prisoners. About 247 $(60 \%)$ of prisoners were sentenced for 2 years and less and $31(7.5 \%)$ prisoners were sentenced for lifelong. From the total participants, 127 (30.9\%) prisoners were reconvicted prisoners. Stealing $(174,42.3 \%)$, murder $(99,24.1 \%)$, and physical assault $(74,18 \%)$ were the most common causes of imprisonment. Eighty-four (20.4\%) prisoners reported that they were punished for their misconduct in their previous incarceration while they were in prison. More than one third of the total respondents $(160,38.9 \%)$ reported that they had a family history of imprisonment and, out of them, 50 (31\%) had psychopathy (see Table 2).

\section{Clinical-Related Factors}

Nearly one fourth of the total respondents $(88,21.4 \%)$ reported that their family members had a history of mental illness and were followed-up in a mental health facility. From the total participants, $30(7.3 \%)$ had treatment and a follow-up history of mental illness. Again, 48 (11.7\%) participants reported they had a chronic medical illness and they were on treatment and on follow-up. Almost one third $(130,31 \%)$ of the participants had a family history of alcohol use and, among them, 89 (68.4\%) had psychopathy (see Table 3).

\section{Environmental-Related Factors}

About half of the respondents $(215,52.3 \%)$ had poor social support and, among them, 49 (23\%) had psychopathy. From the total participants, 265 (64.5\%) had experienced two or more traumatic life events in their lifetime and, among them, 74 (28\%) had psychopathy. Regarding the childhood family conflict, nearly one fourth (97, 23.6\%) had experienced childhood family conflict. Concerning maltreatment, about one third of the respondents $(123,29.9 \%)$ had multiple childhood maltreatment, and among them $29(23.6 \%)$ had psychopathy (see Table 4).

\section{Substance-Related Factors}

Half of the respondents $(209,50.9 \%)$ ever used khat and, among them, 65 (31\%) had psychopathy. About half of respondents, 203 (49. 4\%) used khat over the past 3 months and, among them, 62 (30.5\%) had psychopathy; and from the total respondents, 152 (37\%) were high risk khat users and, from high risk khat users, 69 (45\%) had psychopathy. Less than one fourth $(86,20.9 \%)$ of the 
Table I Socio-Demographic and Economic Characteristics of Respondents Among Newly-Admitted Prisoners of Bench Sheko and West Omo Zone Correction Center, Mizan Aman Ethiopia, 2019 (N=4II)

\begin{tabular}{|c|c|c|c|c|}
\hline \multirow[t]{2}{*}{ Variables } & \multicolumn{2}{|c|}{ Psychopathy } & \multirow{2}{*}{$\begin{array}{l}\text { Frequency } \\
\text { No }\end{array}$} & \multirow{2}{*}{$\begin{array}{l}\text { Percentage } \\
\%\end{array}$} \\
\hline & Yes (\%) & No (\%) & & \\
\hline \multicolumn{5}{|l|}{ Age } \\
\hline $18-24$ & $50(37.50)$ & $83(62.5)$ & 133 & 32.4 \\
\hline $25-44$ & $29(12)$ & $220(88)$ & 249 & 60.4 \\
\hline$\geq 45$ & $21(72)$ & $8(28)$ & 29 & 7.1 \\
\hline \multicolumn{5}{|l|}{ Occupation } \\
\hline Farmer & 31 (I4.7) & I79 (85.3) & 210 & 51.1 \\
\hline Unemployed & $6(37.5)$ & $10(62.5)$ & 16 & 3.9 \\
\hline Daily laborer & $13(15.6)$ & $70(84.4)$ & 83 & 20.2 \\
\hline Student & $8(24.2)$ & $25(75.8)$ & 33 & 8 \\
\hline Employed & $16(76)$ & $5(23.4)$ & 21 & 5.1 \\
\hline Driver & $17(68)$ & $8(32)$ & 25 & 6.1 \\
\hline Others & 9 (39) & $14(60.1)$ & 23 & 5.6 \\
\hline \multicolumn{5}{|l|}{ Ethnicity } \\
\hline Bench & - & - & 208 & 50.6 \\
\hline Sheko & - & - & 63 & 15.3 \\
\hline Amara & - & - & 53 & 12.9 \\
\hline Kefa & - & - & 32 & 7.8 \\
\hline Dizi & - & - & 17 & 4.1 \\
\hline Mnit & - & - & 15 & 3.6 \\
\hline Others & - & - & 23 & 5.6 \\
\hline \multicolumn{5}{|l|}{ Sex } \\
\hline Male & $96(24)$ & $308(76)$ & 404 & 98.3 \\
\hline Female & $4(57.2)$ & $3(42.8)$ & 7 & 1.7 \\
\hline \multicolumn{5}{|l|}{ Income } \\
\hline$<1,200$ & $25(39)$ & $39(61)$ & 64 & 15.6 \\
\hline$\geq 1,200$ & $75(21.6)$ & $272(78.4)$ & 347 & 84.4 \\
\hline \multicolumn{5}{|l|}{ Marital status } \\
\hline Married & $14(6)$ & $213(94)$ & 227 & 55.2 \\
\hline Single & $50(47.6)$ & $55(52.4)$ & 105 & 25.5 \\
\hline Divorced & $19(42.2)$ & $26(57.8)$ & 45 & 10.9 \\
\hline Separated & $15(5 \mid .2)$ & $14(48.3)$ & 29 & 7.2 \\
\hline Widowed & $2(40)$ & $3(60)$ & 5 & 1.2 \\
\hline \multicolumn{5}{|l|}{ Religion } \\
\hline Protestant & - & - & 268 & 65.2 \\
\hline Orthodox & - & - & 135 & 32.5 \\
\hline Muslim & - & - & 7 & 1.7 \\
\hline Kalicha & - & - & 1 & 0.2 \\
\hline \multicolumn{5}{|l|}{ Education } \\
\hline Primary & $38(14)$ & $234(86)$ & 273 & 66.4 \\
\hline Secondary and above & $58(47)$ & $66(53)$ & 124 & 30.1 \\
\hline Unable to read and write & $4(28.5)$ & $10(71.5)$ & 14 & 3.4 \\
\hline
\end{tabular}

participants used tobacco over the past 3 months and from the total respondents, $56(13.6 \%)$ were high risk tobacco users. Around one fourth, $91(22.1 \%)$ of the participants used alcohol over the past 3 months and from the total respondents, 65 (15.8\%) were high risk alcohol users (see Table 5). 
Table 2 Criminal and Prison Characteristics of Respondents Among Newly-Admitted Prisoners of Bench Sheko and West Omo Zone Correction Center Mizan Aman, Ethiopia, 2019 ( $N=4 \mathrm{II})$

\begin{tabular}{|c|c|c|c|c|}
\hline \multirow[t]{2}{*}{ Variables } & \multicolumn{2}{|c|}{ Psychopathy } & \multirow{2}{*}{$\begin{array}{l}\text { Frequency } \\
\text { No }\end{array}$} & \multirow{2}{*}{$\begin{array}{l}\text { Percentage } \\
\%\end{array}$} \\
\hline & Yes (\%) & No (\%) & & \\
\hline \multicolumn{5}{|l|}{ Prior prison } \\
\hline Once & $2(2.7)$ & $71(97.3)$ & 72 & 17.8 \\
\hline Twice & $12(28.6)$ & $30(71.4)$ & 42 & 10.2 \\
\hline 3 and above & $12(100)$ & $0(0)$ & 12 & 2.9 \\
\hline No & $4(26)$ & $210(74)$ & 284 & 69.1 \\
\hline \multicolumn{5}{|l|}{ Crime type } \\
\hline Stealing & $7(4)$ & $167(96)$ & 174 & 42.3 \\
\hline Fighting & $20(27)$ & $54(73)$ & 74 & 18 \\
\hline Rape & $2(35)$ & $15(65)$ & 17 & 4.1 \\
\hline Sexual assault & $4(2 I)$ & $15(79)$ & 19 & 4.6 \\
\hline Homicide & $60(60.6)$ & 39 (39.4) & 99 & 24.1 \\
\hline Robbery & $3(23)$ & $10(77)$ & 13 & 3.2 \\
\hline Dual marriage & I (14.3) & $6(85.7)$ & 7 & 1.7 \\
\hline Others & $3(37.5)$ & $5(62.5)$ & 8 & 1.9 \\
\hline \multicolumn{5}{|l|}{ Reconviction } \\
\hline Yes & $88(69)$ & $39(3 \mathrm{I})$ & 127 & 30.9 \\
\hline No & $12(4.2)$ & $272(95.8)$ & 327 & 69.1 \\
\hline \multicolumn{5}{|c|}{ Misconduct in prior prison } \\
\hline Yes & $69(82)$ & $15(18)$ & 84 & 20.4 \\
\hline No & $31(9.5)$ & $296(90.5)$ & 347 & 79.6 \\
\hline \multicolumn{5}{|c|}{ Family history of prison } \\
\hline Yes & $50(3 I)$ & $110(69)$ & 160 & 38.9 \\
\hline No & $50(19.9)$ & $201(80.1)$ & 251 & 61.9 \\
\hline \multicolumn{5}{|l|}{ Set fire } \\
\hline Yes & $3(75)$ & I (25) & 4 & I \\
\hline No & $97(23.8)$ & $310(76.2)$ & 407 & 99 \\
\hline \multicolumn{5}{|l|}{ Killed animals } \\
\hline Yes & $2(50)$ & $2(50)$ & 4 & I \\
\hline No & $99(24)$ & $308(76)$ & 407 & 99 \\
\hline
\end{tabular}

Note: Other crimes include; deforestation, illegal land ownership, and political issues.

\section{Factors Associated with Psychopathy}

The bivariate analysis showed; Age, occupation, crime, family history of mental illness, reconviction, having mental illness, family history of alcohol use, high risk khat use, high risk tobacco use, childhood maltreatment, family history of prison, family history of conflict, traumatic life event, and social support were significantly associated variables with psychopathy. However, multivariable logistic regression showed that six variables were positively associated with psychopathy: childhood maltreatment (AOR $=6$., 95\% $\mathrm{CI}=2.2-17.5)$, risky khat use ( $\mathrm{AOR}=4.6,95 \% \mathrm{CI}=2.4-8.7)$, poor social support (AOR=3.5, 95\% $\mathrm{CI}=1.9-6.6)$, a family history of imprisonment ( $\mathrm{AOR}=3 ., 95 \% \mathrm{CI}=1.5-6$ ), a history of trauma $(\mathrm{AOR}=2.3,95 \% \mathrm{CI}=1.1-4.8)$, and reoffending (AOR=2., 95\% CI=1.1-3.8) (see Table 6).

High risk khat users were more than 4-times more likely to have psychopathy than non-risk khat users ( $\mathrm{AOR}=4.6,95 \% \mathrm{CI}=2.4-8.7$ ). Also, prisoners with poor social support were more than 3-times more likely to have psychopathy than prisoners with good social support ( $\mathrm{AOR}=3.5,95 \% \mathrm{CI}=1.9-6.6$ ). Prisoners who were convicted at least once before were 2-times more likely to have psychopathy than prisoners with no prior prison history $(\mathrm{AOR}=2,95 \% \mathrm{CI}=1.1-3.8)$. Prisoners who had 
Table 3 Clinical Characteristics of Respondents Among Newly-Admitted Prisoners of Bench Sheko and West Omo Zone Correction Center Mizan Aman Ethiopia (N=4II), 2019

\begin{tabular}{|c|c|c|c|c|}
\hline \multirow[t]{2}{*}{ Variables } & \multicolumn{2}{|c|}{ Psychopathy } & \multirow{2}{*}{$\begin{array}{l}\text { Frequency } \\
\text { No }\end{array}$} & \multirow{2}{*}{$\begin{array}{l}\text { Percentage } \\
\%\end{array}$} \\
\hline & Yes (\%) & No (\%) & & \\
\hline \multicolumn{5}{|l|}{ History of mental illness } \\
\hline Yes & $20(66.6)$ & $10(34.4)$ & 30 & 7.3 \\
\hline No & $80(21)$ & 301 (79) & 381 & 92.7 \\
\hline \multicolumn{5}{|l|}{ Chronic physical illness } \\
\hline Yes & $28(58.3)$ & $20(4 I .7)$ & 48 & 11.7 \\
\hline No & $72(20)$ & $291(80)$ & 363 & 88.3 \\
\hline \multicolumn{5}{|l|}{ Psychopathy treatment } \\
\hline Yes & $8(88.9)$ & I (II.I) & 9 & 2.2 \\
\hline No & $92(22.8)$ & $310(77.2)$ & 402 & 97.8 \\
\hline \multicolumn{5}{|l|}{ Mental illness of family } \\
\hline Yes & $67(76)$ & $21(24)$ & 88 & 21.4 \\
\hline No & $33(10)$ & $290(90)$ & 323 & 78.6 \\
\hline \multicolumn{5}{|l|}{ Family history of alcohol } \\
\hline Yes & $89(68.4)$ & $4 \mid(3 \mid .6)$ & 130 & 31.6 \\
\hline No & II (5) & $210(95)$ & 281 & 68.4 \\
\hline
\end{tabular}

Table 4 Environmental Characteristics of Respondents Among Newly-Admitted Prisoners of Bench Sheko and West Omo Zone Correction Center Mizan Aman Ethiopia (N=4II), 2019

\begin{tabular}{|c|c|c|c|c|}
\hline \multirow[t]{2}{*}{ Variables } & \multicolumn{2}{|c|}{ Psychopathy } & \multirow{2}{*}{$\begin{array}{l}\text { Frequency } \\
\text { No }\end{array}$} & \multirow{2}{*}{$\begin{array}{l}\text { Percentage } \\
\%\end{array}$} \\
\hline & Yes (\%) & No (\%) & & \\
\hline \multicolumn{5}{|l|}{ Social support } \\
\hline Poor & $49(23)$ & $166(76)$ & 215 & 52.3 \\
\hline Moderate & $3(5.6)$ & $50(94.4)$ & 53 & 12.9 \\
\hline Good & $48(33.6)$ & $95(66.4)$ & 143 & 34.8 \\
\hline \multicolumn{5}{|l|}{ Traumatic life event } \\
\hline Multiple & $74(28)$ & 191 (72) & 265 & 64.5 \\
\hline Only one & $5(17.8)$ & $23(82.2)$ & 28 & 6.8 \\
\hline No trauma exposure & $21(17.8)$ & $97(82.2)$ & 118 & 28.7 \\
\hline \multicolumn{5}{|l|}{ Childhood maltreatment } \\
\hline Multiple & $29(23.6)$ & $94(76.4)$ & 123 & 29.9 \\
\hline Only 2 & $57(35)$ & $105(65)$ & 162 & 39.4 \\
\hline Only I & $3(25)$ & $9(75)$ & 12 & 2.9 \\
\hline No & II (I0) & $103(90)$ & 114 & 27.7 \\
\hline \multicolumn{5}{|l|}{ Excessive television watch } \\
\hline Yes & $9(60)$ & $6(40)$ & 15 & 3.6 \\
\hline No & $91(23)$ & $305(77)$ & 396 & 96.4 \\
\hline \multicolumn{5}{|l|}{ Family history of conflict } \\
\hline Yes & $61(63)$ & $36(37)$ & 97 & 23.6 \\
\hline No & $39(12)$ & $275(88)$ & 314 & 76.4 \\
\hline
\end{tabular}


Table 5 Substance Characteristics of Respondents Among Newly-Admitted Prisoners of Bench Sheko and West Omo Zone Correction Center Mizan Aman Ethiopia (N=4II), 2019

\begin{tabular}{|c|c|c|c|c|}
\hline \multirow{2}{*}{ Variables } & \multicolumn{2}{|c|}{ Psychopathy } & \multirow{2}{*}{$\begin{array}{l}\text { Frequency } \\
\text { No }\end{array}$} & \multirow{2}{*}{$\begin{array}{l}\text { Percentage } \\
\%\end{array}$} \\
\hline & Yes (\%) & No (\%) & & \\
\hline \multicolumn{5}{|l|}{ Risky khat use } \\
\hline High risk & $69(45)$ & $83(55)$ & 152 & 37 \\
\hline Moderate risk & $8(47)$ & $9(53)$ & 17 & 4.1 \\
\hline Low risk & $15(42.3)$ & $20(57)$ & 40 & 8.1 \\
\hline Non-risk & $8(3.8)$ & $199(96.2)$ & 207 & 50.1 \\
\hline \multicolumn{5}{|l|}{ Risky tobacco use } \\
\hline High risk & $40(71.4)$ & $16(28.6)$ & 56 & 13.6 \\
\hline Moderate risk & $15(75)$ & $5(25)$ & 20 & 4.9 \\
\hline Low risk & $7(70)$ & $3(30)$ & 10 & 2.4 \\
\hline Non-risk & 38 (11.7) & $287(88.3)$ & 325 & 79.1 \\
\hline \multicolumn{5}{|l|}{ Risky alcohol use } \\
\hline High risk & 47 (7I.2) & $19(28.8)$ & 66 & 16.1 \\
\hline Moderate risk & I (50) & $\mathrm{I}(50)$ & 2 & 0.5 \\
\hline Low risk & $12(50)$ & $12(50)$ & 24 & 5.8 \\
\hline Non-risk & $40(12.5)$ & $279(87.5)$ & 319 & 77.6 \\
\hline \multicolumn{5}{|l|}{ Current khat use } \\
\hline Yes & $62(30.5)$ & |4| (69.5) & 203 & 49.4 \\
\hline No & $38(18.2)$ & $170(82.80)$ & 208 & 50.6 \\
\hline \multicolumn{5}{|c|}{ Current tobacco use } \\
\hline Yes & $62(72)$ & $24(28)$ & 86 & 20.9 \\
\hline No & 38 (11.7) & $287(88.3)$ & 325 & 79.1 \\
\hline \multicolumn{5}{|c|}{ Current alcohol use } \\
\hline Yes & $59(65)$ & $32(35)$ & 91 & 22.1 \\
\hline No & $4 \mid(12.8)$ & $279(87.2)$ & 320 & 77.9 \\
\hline \multicolumn{5}{|l|}{ Ever khat use } \\
\hline Yes & $65(31)$ & I44 (69) & 209 & 50.9 \\
\hline No & $35(17.3)$ & 167 (82.7) & 202 & 49.1 \\
\hline \multicolumn{5}{|l|}{ Ever tobacco use } \\
\hline Yes & $62(72.9)$ & $23(27.1)$ & 85 & 20.7 \\
\hline No & 38 (II.6) & $288(88.4)$ & 326 & 79.3 \\
\hline \multicolumn{5}{|l|}{ Ever alcohol use } \\
\hline Yes & $59(64.8)$ & $32(35)$ & 91 & 22.1 \\
\hline No & 41 (12.9) & $279(87.1)$ & 320 & 77.9 \\
\hline
\end{tabular}

multiple childhood maltreatment were nearly 6-times more likely to develop psychopathy than prisoners without childhood maltreatment $(\mathrm{AOR}=6,95 \% \quad \mathrm{CI}=2.2-17.5)$.

Prisoners with a family history of prison were about 3-times more likely to develop psychopathy than prisoners without a family history of prison $(\mathrm{AOR}=3,95 \% \mathrm{CI}=1.5-$ $6)$. In addition, prisoners who had exposure to multiple traumatic life events were more than 2-times more likely to have psychopathy than prisoners with no traumatic life event exposure $(\mathrm{AOR}=2.3,95 \% \mathrm{CI}=1.1-4.8)$ (see Table 6$)$.

\section{Discussion}

This cross-sectional study assessed the prevalence of psychopathy among newly-admitted prisoners of Bench Sheko and West Omo zone correction center and its associated factors. In this study, the prevalence of psychopathy 
Table 6 Bivariate and Multivariate Logistic Regression Analysis for Independent Predictors of Psychopathy Among Newly-Admitted Prisoners of Bench Sheko and West Omo Zone Correction Center Mizan Aman, Ethiopia (N=4II), 2019

\begin{tabular}{|c|c|c|c|c|}
\hline \multirow[t]{2}{*}{ Variables } & \multicolumn{2}{|c|}{ Psychopathy } & \multirow[t]{2}{*}{ COR at $95 \% \mathrm{Cl}$} & \multirow[t]{2}{*}{ AOR at $95 \% \mathrm{Cl}$} \\
\hline & Yes (\%) & No (\%) & & \\
\hline \multicolumn{5}{|l|}{ Age } \\
\hline $18-24$ & $50(37.50)$ & $83(62.5)$ & $0.23(0.9-0.55)$ & $0.5(0.1 I-2.1)$ \\
\hline $25-44$ & $29(12)$ & $220(88)$ & $0.26(0.11-0.60)$ & $0.8(0.2-3.12)$ \\
\hline$\geq 45$ & $21(72)$ & $8(28)$ & 1 & 1 \\
\hline \multicolumn{5}{|l|}{ Occupation } \\
\hline Farmer & $31(14.7)$ & $179(85.3)$ & $0.8(0.3-1.92)$ & $0.6(0.15 .21)$ \\
\hline Unemployed & $6(37.5)$ & $10(62.5)$ & $0.9(2-3.3)$ & $1.6(0.7-12)$ \\
\hline Daily laborer & $13(15.6)$ & $70(84.4)$ & I.6 (0.6-4.3) & $0.9(0.2 .-3.7)$ \\
\hline Student & $8(24.2)$ & $25(75.8)$ & $0.5(I-1.58)$ & $0.8(0.16-4.4)$ \\
\hline Employed & $16(76)$ & $5(23.4)$ & $4.9(0.1-18.1)$ & $3(0.45-20)$ \\
\hline Driver & $17(68)$ & $8(32)$ & $3.3(1-10.3)$ & $2(0.3-12)$ \\
\hline Others & $9(39)$ & $14(60.1)$ & 1 & 1 \\
\hline \multicolumn{5}{|l|}{ Social support } \\
\hline Poor & $49(23)$ & $166(76)$ & $2.5(1.6-3.8)$ & $3.5(1.9-6.6)$ \\
\hline Medium & $3(5.6)$ & $50(94.4)$ & $0.11(0.03-0.4)$ & $0.2(0.04-0.94)$ \\
\hline Good & $48(33.6)$ & $95(66.4)$ & 1 & I \\
\hline \multicolumn{5}{|c|}{ Family mental illness } \\
\hline Yes & $67(76)$ & $21(24)$ & $0.15(0.08-0.3)$ & I.3 (0.5-3.2) \\
\hline No & $33(10)$ & $290(90)$ & 1 & 1 \\
\hline \multicolumn{5}{|c|}{ History of mental illness } \\
\hline Yes & $20(66.6)$ & $10(34.4)$ & $0.33(0.15-0.75)$ & I $(0.3-3.67)$ \\
\hline No & $80(2 I)$ & $301(79)$ & 1 & 1 \\
\hline \multicolumn{5}{|c|}{ Traumatic life event } \\
\hline Multiple & $74(28)$ & $191(72)$ & $5.5(3.2-9.4)$ & $2.3(I . I-4.8)$ \\
\hline I trauma & $5(17.8)$ & $23(82.2)$ & I (0.3-2.9) & I.5 (0.39-6.0) \\
\hline No trauma & $21(17.8)$ & $97(82.2)$ & $\mathrm{I}$ & I \\
\hline \multicolumn{5}{|l|}{ Reconviction } \\
\hline Yes & $88(69)$ & $39(3 I)$ & $5.4(3.4-8.6)$ & $2(1.1-3.8)$ \\
\hline No & $12(4.2)$ & $272(95.8)$ & 1 & I \\
\hline \multicolumn{5}{|c|}{ Family history of alcohol } \\
\hline Yes & $89(68.4)$ & $4 I(31.6)$ & $5.2(3.3-8.2)$ & $1.7(0.85-3.7)$ \\
\hline No & II (5) & $210(95)$ & 1 & 1 \\
\hline \multicolumn{5}{|c|}{ Family history of prison } \\
\hline Yes & $50(3 I)$ & $110(69)$ & $12(6.1-23.3)$ & $3(1.5-6.0)$ \\
\hline No & 50 (19.9) & $201(80.1)$ & 1 & I \\
\hline \multicolumn{5}{|c|}{ Childhood maltreatment } \\
\hline Multiple & $29(23.6)$ & $94(76.4)$ & $12(6.1-23)$ & $6(2.2-17.6)$ \\
\hline Only 2 & $57(35)$ & $105(65)$ & $3.5(0.8-15.2)$ & $1.9(0.8-4.6)$ \\
\hline Only I & $3(25)$ & $9(75)$ & $3.1(0.7-13.4)$ & I.4 (0.27-7.4) \\
\hline No & II (9.6) & $103(90.36)$ & 1 & 1 \\
\hline \multicolumn{5}{|l|}{ Risky khat use } \\
\hline High risk & $69(45)$ & $83(55)$ & $11.6(7.1-19.2)$ & $4.6(2.4-8.7)$ \\
\hline Moderate risk & $8(47)$ & $9(53)$ & $3.9(1.4-10.9)$ & $3.2(0.87-11.6)$ \\
\hline
\end{tabular}

(Continued) 
Table 6 (Continued).

\begin{tabular}{|c|c|c|c|c|}
\hline \multirow[t]{2}{*}{ Variables } & \multicolumn{2}{|c|}{ Psychopathy } & \multirow[t]{2}{*}{ COR at $95 \% \mathrm{Cl}$} & \multirow[t]{2}{*}{ AOR at $95 \% \mathrm{Cl}$} \\
\hline & Yes (\%) & No (\%) & & \\
\hline Low risk & $15(42.3)$ & $20(57)$ & $3.33(1.5-7.1)$ & $1.5(0.6-3.8)$ \\
\hline Non-risk & $8(3.8)$ & $199(96.2)$ & I & 1 \\
\hline \multicolumn{5}{|l|}{ Risky tobacco use } \\
\hline High risk & $40(7 \mid .4)$ & $16(28.6)$ & $4.9(2.6-9.2)$ & $0.5(0.2-1.6)$ \\
\hline Moderate risk & $15(75)$ & $5(25)$ & $5.9(2-9.16 .8)$ & $0.6(0.1-3.2)$ \\
\hline Low risk & $7(70)$ & $3(30)$ & $4.6(1-18)$ & $1.3(0.2-7.1)$ \\
\hline Non-risk & 38 (II.7) & $287(88.3)$ & 1 & I \\
\hline \multicolumn{5}{|c|}{ History of family conflict } \\
\hline Yes & $61(63)$ & $36(37)$ & $0.32(0.22-0.5 \mathrm{I})$ & $2(I-4)$ \\
\hline No & $39(12)$ & $275(88)$ & I & I \\
\hline \multicolumn{5}{|l|}{ Crime type } \\
\hline Stealing & $7(4)$ & $167(96)$ & I $(0.2-4.5)$ & $68(0.3-1.6)$ \\
\hline Fighting & $20(27)$ & $54(73)$ & $0.6(0.1-2.6)$ & $0.3(0.04-1.7)$ \\
\hline Rape & $2(35)$ & $15(65)$ & $0.9(1.5-5.1)$ & I.I (0.3-4.22) \\
\hline Sexual assault & $4(2 I)$ & $15(79)$ & $0.44(0.07-2.7)$ & $2.1(1-4.5)$ \\
\hline Homicide & $60(60.6)$ & $39(39.4)$ & $3.5(0.7-15)$ & $0.5(0.09-2.85)$ \\
\hline Robbery & $3(23)$ & $10(77)$ & $0.5(0.07-3.4)$ & $0.24(0.02-2.8)$ \\
\hline Dual marriage & I (I4.3) & $6(85.7)$ & $0.3(0.02-3.5)$ & I.I (0.08-13) \\
\hline Others & $3(37.5)$ & $5(62.5)$ & 1 & I \\
\hline
\end{tabular}

Note: $\mathrm{I}=$ reference category.

was $24.3 \%$. This finding is similar with a studies done in the US, ${ }^{28,29}$ and South Florida, ${ }^{17}$ and higher than studies done in China, ${ }^{12}$ Germany, ${ }^{30}$ and England and Wales. ${ }^{31}$ The difference might be due to; sociocultural difference, other studies were not on newly admitted prisoners, tool difference, and study design difference.

High risk khat users were about 4-times more likely to have psychopathy than non-risk khat users. This finding was in line with studies done in Nigeria, ${ }^{32}$ England and Wales, ${ }^{31}$ the University of South Dakota, US, ${ }^{33}$ University of Otego, Wellington, New Zealand, ${ }^{34}$ Sweden, ${ }^{35}$ and also astudy done on Lowa, US, ${ }^{36}$ where substance abuse is strongly and positively associated with psychopathy. The reason might be due to people with psychopathy being impulsive and trying new events like substance use despite its consequences. ${ }^{37}$ Another likely reason might be that prisoners with psychopathy use khat in Ethiopia, which is a locally accessible amphetamine type stimulant, in order to get relief from symptoms of aggression; or khat use itself might lead to symptoms of psychopathy. However, it is difficult to find the cause and effect association.

In this study, prisoners with trauma exposure were more significantly and positively associated with psychopathy than prisoners with no history of trauma. This finding was the same as in studies done in Germany, ${ }^{38}$ New York, ${ }^{39}$ and South Florida. ${ }^{40}$ The main reason might be due to people with psychopathy being vulnerable to traumatic injuries, accidents, homicide, and suicide attempts, as a result of their impulsive, aggressive, and risky behaviors, and they may repeatedly disregard and jeopardize their own safety and the safety of others and place themselves and others in danger. ${ }^{41}$

Being reconvicted had higher odds to be screened positive for psychopathy when compared with their counterparts. This finding was in line with a similar study done in China, ${ }^{12}$ Canada, ${ }^{20}$ and Chicago, ${ }^{21}$ where psychopathy is more common in those who have repeated admission to prison. The reason might be due to people with psychopathy frequently performing acts that result in arrest and they are unable to conform to social norms as well as their impulsion to commit crime is genetically oriented. ${ }^{3}$

In this study, having poor social support had a higher odds of developing psychopathy than prisoners with good social support, and also having moderate social support was protective against psychopathy. The studies done among non-institutionalized, young, adult, psychopathic participants in Japan ${ }^{42}$ and institutionalized women prisoners in Indonesia ${ }^{16}$ revealed that lack of social support was 
significantly and positively associated with psychopathy. The reason might be due to lack of any criticisms from a nearby person, so that they continue their unacceptable behaviors. Another reason might be since they have socially deviant behavior; they can have significant difficulties in maintaining stable social relationship to fulfill their social and financial obligations. Moreover, it might be due to a lack of someone who helps them when they were in problems and a lack of someone who is rewarding and punishing them when they show socially acceptable and unacceptable behaviors, respectively.

Prisoners who had multiple childhood maltreatment were 6-times more likely to develop psychopathy than prisoners without childhood maltreatment. This finding was the same as the study done in the US. $^{6}$ The main reason might be due to childhood and adolescent large stress reactions being harmful, and it reduces stress reactivity and enhances antisocial behavior. ${ }^{43}$ Moreover, psychopathy is the result of their social and cultural experiences of a person in childhood and adolescence encompassing their family dynamics, peer influences, and social values. ${ }^{11}$

Prisoners with a family history of prison were 3-times more likely to develop psychopathy than prisoners with no family history. This study was in line with a similar study done in Florida ${ }^{44}$ and Finland. ${ }^{9}$ The reason could be due to psychopathy being caused by a combination and interaction of genetic and environmental influences. Genetically, it is the intrinsic temperamental tendencies as determined by their genetically influenced physiology and it runs around the family. ${ }^{45}$

Modern psychiatric care among communities in Ethiopia is highly affected by the deep rooted cultural belief that mental illness is because of evil spirit, problems of access to facilities in remote areas, and stigma of people with mental illness. There is a single mental hospital serving about 110 million people in the capital city of Ethiopia. Therefore, the main preference of treatment is holy water and traditional rituals, rather than seeking modern psychiatric care. That is why prisoners have no mental health care facility (clinicians) in prison institutions, and no one stands for psychopathic (mentally ill) prisoners while their case is being seen in court, despite most of their criminal cases being because of their mental illness. In Ethiopia, psychopathic individuals were known by isolation from community members due to their antisocial behaviors, repeated admission to prison centers, and stigma among ethnic groups, and some them were taken to holy water and cultural treatments. Our finding is a good opportunity for screening and providing intervention among people with psychopathy and recognizing ways to prevent reoffending, substance use, trauma exposure, and childhood maltreatment in a prison institution throughout the country.

Psychopathic people are difficult to treat and require a high amount of clinician's effort (attention, time, and money). ${ }^{46}$ Furthermore, people with psychopathy infrequently present for treatment willingly. Prison settings represent an ideal opportunity to administer clinical interventions (group and individual psychotherapy) in people with psychopathy, even though their psychopathic behaviors like lack of empathy, an incapability to form close personal relationships, and manipulative and callous behaviors, are obstacles to effective therapeutic interventions. ${ }^{47,48}$

Future research should be designed to explore more about neurobiological correlates and psychosocial factors that build up for the development of psychopathy. Additionally, the relationship between substance misuse, offending, trauma, and psychopathy need to be understood by using more advanced study designs and a new screening tool for psychopathy.

\section{Limitations of the Study}

Interviewer bias, recall bias, and underreport and denial of criminal activities and substance use. The study has included only a few women; so it is difficult to generalize for female offenders. Prisoners' mental and medical illness were not confirmed by medical record. This study is on newly admitted prisoners and may not be generalized to prisoners who stayed in the prison. In addition, the psychopathy diagnosis was based on interview and file review and it lacks collateral information. Moreover, the PCL-R screening tool was not validated in an Ethiopian context.

\section{Conclusion}

This study has found a high prevalence of psychopathy among newly-admitted prisoners of Bench Sheko and West Omo zone correction center. Reconviction, high risk khat use, family history of prison, childhood maltreatment, traumatic life event, and poor social support were positively associated with psychopathy. In general, despite the high prevalence of psychopathy and its negative consequences, prisoners did not report any treatment given in a prison institution as well as in a community set up. Based on this finding, we recommend prison 
administrators, policy makers, and non-governmental organizations who are working on prison concerns to establish mental health services at correctional centers. Moreover, integrated efforts involving relevant stakeholders are needed to design strategies for early screening of psychopathy to prevent reoffending, and management of risky khat use at admission is crucial.

\section{Abbreviations}

AOR, adjusted odds ratio; ASSIST, Alcohol, Smoking and Substance Involvement Screening Test; CI, confidence Interval; COR, crude odds ratio; JU, Jimma University; PCL-R, Psychopathic Checklist Revised; SPSS, Statistical Package for Social Science; US, United States; WHO, World Health Organization.

\section{Data Sharing Statement}

The data set is obtainable from the corresponding author upon reasonable request.

\section{Ethics Approval and Consent to Participate}

This study was conducted in accordance with the Declaration of Helsinki. Ethical clearance was obtained from the Review Board of Jimma University. The purpose of the study was explained to the study participants. Permission was obtained from prison administration. Written consent was obtained from all study participants before the interview. Participation in the study was completely voluntary, and the right to withdraw from the interview was also secured. Confidentiality of the information was ensured throughout the study.

\section{Acknowledgments}

The authors are very thankful for Jimma University, the study participants, data collectors and supervisors.

\section{Author Contributions}

All authors contributed to the data analysis, drafting or revising the article, have approved the journal to which the article will be submitted, gave final approval of the version to be published, and agree to be responsible for all aspects of the work.

\section{Funding}

This study was funded by Jimma University.

\section{Disclosure}

The authors report no conflicts of interest in this work.

\section{References}

1. Lykken DT. Psychopathy, sociopath, and antisocial personality disorder. Handbook Psychopath. 2018;23:22.

2. Hare RD. Manual for the revised psychopathy checklist, second edition, multi health systems,Toronto. 2003

3. Walsh Z, Allen LC, Kosson DS. Beyond social deviance: substance use disorders and the dimensions of psychopathy. J Pers Disord. 2007;21(3):273-288. doi:10.1521/pedi.2007.21.3.273

4. Sophie Mann J, Lewis M, Ozanne R, McNeill K, Carol A, Ireland CA. Psychopathy and trauma: exploring a potential association. J Int Law Psychiatr. 2020;69:101543. doi:10.1016/j. ijlp.2020.101543

5. Pollock PH. When the killer suffers Post-traumatic stress reactions following Homicide. Legal Criminolog Psychol. 1999;4(2):185-202. doi: $10.1348 / 135532599167842$

6. Weiler BL, Widom CS. Psychopathy and violent behavior in abused and neglected young adults. Crim Behav Ment Health. 1996;6 (3):253-271. doi:10.1002/cbm.99

7. Hemphill JF, Hare RD, Wong S. Psychopathy and recidivism. Review Legal Criminolog Psychol. 1998;3(1):139-170. doi:10.1111/j.20448333.1998.tb00355.x

8. Leistico AM, Salekin RT, DeCoster J, Rogers R. A large-scale meta-analysis relating the Hare measures of psychopathy to antisocial conduct. Law Hum Behav. 2008;32(1):28-45. doi:10.1007/s10979007-9096-6

9. Steadman HJ, Silver E, Monahan J, et al. A classification tree approach to the development of actuarial violence risk assessment tools. Law Hum Behav. 2000;24(1):83. doi:10.1023/ a: 1005478820425

10. Fox B, Bozzay M, Verona E. An analysis of monoamine-related genotypes and childhood trauma in relation to psychopathic traits in men and women. Crime Delinquency. 2020;66:1438-1469. doi: $10.1177 / 0011128720937295$

11. Dolan M. The neuropsychology of prefrontal function in antisocial personality disordered offenders with varying degrees of psychopathy. Psychol Med. 2011;42(8):1715-1725. doi:10.1017/ S0033291711002686

12. Wang MC, Gong J, Gao Y, Zhang X, Yang W, Luo J. Variants of psychopathy in Chinese male offenders: a latent profile analysis in a large prison sample. J Crim Justice. 2020;69:101708. doi:10.1016/j. jcrimjus.2020.101708

13. Thomson ND, Moeller FG, Amstadter AB, Svikis D, Perera RA, Bjork JM. The impact of parental incarceration on psychopathy, crime, and prison violence in women. Int $J$ Offender Ther Comp Criminol. 2020;64(10-11):0306624X20904695.

14. Thomson ND, Vassileva J, Kiehl K. Which features of psychopathy and impulsivity matter most for prison violence? New evidence among female prisoners. Int $J$ Law Psychiatry. 2019;64:26-33. doi:10.1016/j.ijlp.2019.01.001

15. Andelia B, Sutatminingsih R, Siregar RH. The influence of social support on psychological well-being on prisoners of class II-A tanjung gusta women's prison. Int J Sci High Technolog. 2020;23 (2):619-621.

16. Baglivio MT, Wolff KT, DeLisi M, Jackowski K. The role of Adverse Childhood Experiences (ACEs) and psychopathic features on juvenile offending criminal careers to age 18. Youth Violence Juv Justice. 2020;1541204020927075.

17. Fox B, DeLisi M. Psychopathic killers: a meta-analytic review of the psychopathy-homicide nexus. Aggress Violent Behav. 2019;44:67-79. doi:10.1016/j.avb.2018.11.005 
18. Coid J, Young M, Ullrich S, Roberts A, Robert DH. Prevalence and correlates of psychopathic traits in the household population of great Britain. Int Law Psychiatry. 2009;32(2):65-73. doi:10.1016/j. ijlp.2009.01.002

19. Porter S, Woodworth M, Earle J, Drugge J, Boer D. Characteristics of sexual homicides committed by psychopathic and no psychopathic offenders. Law Hum Behav. 2003;27(5):459-470. doi:10.1023/ A:1025461421791

20. Serin RC, Amos NL. The role of psychopathy in the assessment of dangerousness. Int $J$ Law Psychiatry. 1995;18(2):231-238. doi:10.1016/0160-2527(95)00008-6

21. Harris GT, Skilling TA, Rice ME. The construct of psychopathy. Crime Justice. 2001;28(4):197-264. doi:10.1086/652211

22. Kosson D. The treatability of psychopathy. Aftermath: surviving psychopathy foundation. 2019.

23. Salekin R, Worley C, Grimes RD. Treatment of psychopathy: a review and brief introduction to the mental model approach for psychopathy. Behav Sci Law. 2010;28:235-266. doi:10.1002/bsl.928

24. Bøen H, Dalgard OS, Bjertness E. The importance of social support in the associations between psychological distress and somatic health problems and socio-economic factors among older adults living at home. BMC Geriatr. 2012;12(1):27. doi:10.1186/1471-2318-12-27

25. Humeniuk R, Ali R, Babor TF. Validation of the alcohol, smoking and substance involvement screening test (ASSIST). Addiction. 2008;103(6):1039-1047. doi:10.1111/j.1360-0443.2007.02114.x

26. Gray MJ, Litz BT, Hsu JL, Lombardo TW. Psychometric properties of the life events checklist. Assessment. 2004;11(4):330-341. doi:10.1177/1073191104269954

27. Meryl S, Alexandra M Screening for Adverse childhood experience. 2019

28. Hare RD. Psychopathy: a clinical and forensic overview. Psychiatry Clin North Am. 2006;29(3):709-724. doi:10.1016/j.psc.2006.04.007

29. Richard P. Psychopathy, sociopath, and antisocial personality disorder. Forensic Res Criminol Int j. 2016;2(2):258-290.

30. Lehmann A, Ittel A. Aggressive behavior and measurement of psychopathy in female inmates of German prisons. Int J Law Psychiatry. 2012;35(3):190-197. doi:10.1016/j.ijlp.2012.02.007

31. Coid J, Yang M, Ullrich S, et al. Psychopathy among prisoners in England and Wales. Int J Law Psychiatry. 2009;32(3):134-141. doi:10.1016/j.ijlp.2009.02.008

32. Maria Chidi C, Ifeagwazi CM, Ugwu D. Psychopathy and aggressive behaviour among Nigerian male prison inmates: the moderating role of substance abuse. J Psychol Afri. 2018;28(5):365370. doi:10.1080/ 14330237.2018.1523342

33. Slade K, Forrester A. Measuring IPDE-SQ personality disorder prevalence in pre-sentence and early-stage prison populations, with sub-type estimates. Int J Law Psychiatry. 2013;36(3-4):207-212. doi:10.1016/j.ijlp.2013.04.018

34. Marco A, Antón JJ, Saiz de la Hoya P, et al. Personality disorders among Spanish prisoners starting hepatitis $\mathrm{C}$ treatment, prevalence and associated factors. Psychiatr Res Prev Assoc Factors. 2015;230 (3):749-756

Psychology Research and Behavior Management
35. Virtanen S, Laativala A, Andershed H. Do psychopathic personality traits in childhood predict subsequent criminality and psychiatric outcomes over and above childhood behavioral disorders. J Crim Justice. 2020;101761. doi:10.1016/j.jcrimjus.2020.101761

36. Black DW, Gunter T, Loveless P, Allen J, Sieleni B. Antisocial personality disorder in incarcerated offenders: psychiatric comorbidity and quality of life. Ann Clin Psychiatry. 2010;22(2):113-120.

37. Macciò A, Romana F, Sisti D, Bruno M, Rocchi L, Rita D. Mental disorders in Italian prisoners: results of the REDiMe study. Psychiatry Res. 2015;225(3):522-530. doi:10.1016/j. psychres.2014.11.053

38. Sevecke K, Franke S, Kosson D, Krischer M. Emotional deregulation and trauma predicting psychopathy dimensions in female and male juvenile offenders. Child Adolesc Psychiatr Ment Health. 2016;10:43. doi:10.1186/s13034-016-0130-7

39. Behaviour C, Howard RC, Mccarthy L, Huband N. Re-offending in forensic patients released from secure care: the role of antisocial/ borderline personality disorder co-morbidity, substance dependence and severe childhood conduct disorder. Criminal Behav Ment Health. 2013;23(3).

40. El-Gilany A, Khater M, Gomaa Z, Hussein EHI. Psychiatric disorders among prisoners: a national study in Egypt. East Asian Arch Psychiatr. 2016;26(1):30-38.

41. Donald W, Black M. The natural history of antisocial personality disorder. Can J Psychiatr. 2015;60(7):309-314. doi:10.1177/ 070674371506000703

42. Masuia K, Fujiwarab H, Ura M. Social exclusion mediates the relationship between psychopathy and aggressive humor style in non-institutionalized young adults. J Crim Justice. 2013;55 (2):180-184.

43. Rivera F, López I, Guarnaccia P, Ramirez R, Canino G, Bird H Perceived discrimination and antisocial behaviors in Puerto Rican Children. J Immigrants Minor Health. 2011;13(461):453. doi:10.1007/s10903-010-9421-x

44. Kevin M, Beaver Meghan W, Rowland Joseph A, Schwartz Joseph L. The genetic origins of psychopathic personality traits in adult males and females: results from an adoption-based study. J Crim Justice. 2011;39(5):426-432. doi:10.1016/j.jcrimjus.2011.07.002

45. Tiihonen J, Rautiainen M-R, Ollila HM, et al. Genetic background of extreme violent behavior. J Mol Psychiatr. 2014;20(6).

46. Van Stelle KR, Blumer C, Moberg DP. Treatment retention of dually diagnosed offenders in an institutional therapeutic community. Behav Sci Law. 2004;22(4):585-597. doi:10.1002/bsl.602

47. Taylor J, Lang AR. Psychopathy and substance use disorders. In: Patrick CJ, editor. Handbook of Psychopathy. 2006:495-511.

48. Harris GT, Rice ME. Treatment of psychopathy. Handbook Psychopat. 2006;555-572.

\section{Publish your work in this journal}

Psychology Research and Behavior Management is an international, peer-reviewed, open access journal focusing on the science of psychology and its application in behavior management to develop improved outcomes in the clinical, educational, sports and business arenas. Specific topics covered in the journal include: Neuroscience, memory and decision making; Behavior modification and management; Clinica applications; Business and sports performance management; Social and developmental studies; Animal studies. The manuscript management system is completely online and includes a very quick and fair peer-review system, which is all easy to use. Visit http://www. dovepress.com/testimonials.php to read real quotes from published authors. 